\title{
Oscillatory behavior of a class of fractional difference equations with damping
}

\author{
A.George Maria Selvam ${ }^{1 *}$, M. Reni Sagayaraj ${ }^{1}$, M.Paul Loganathan ${ }^{2}$ \\ ${ }^{1}$ Sacred Heart College,Tirupattur - 635 601, S. India \\ ${ }^{2}$ Departments of Mathematics, Dravidian University, Kuppam \\ *Corresponding author E-mail: agmshc@gmail.com
}

Copyright $\odot 2014$ Selvam et al. This is an open access article distributed under the Creative Commons Attribution License, which permits unrestricted use, distribution, and reproduction in any medium, provided the original work is properly cited.

\section{Abstract}

This paper investigates the oscillation of a class of fractional difference equations with damping term of the form $\Delta\left(c(t)\left(\Delta^{\alpha} x(t)\right)^{\gamma}\right)+p(t)\left(\Delta^{\alpha} x(t)\right)^{\gamma}+q(t)\left(\sum_{s=t}^{t-1+\alpha}(t-s-1)^{(-\alpha)} x(s)\right)^{\gamma}=0, t \in N_{t_{0}+1-\alpha}$

where $\Delta^{\alpha}$ denotes the Riemann-Liouville difference operator of order $0<\alpha \leq 1$ and $\gamma>0$ is a quotient of odd positive integers. Based on a generalized Riccati transformation and some inequalities, we establish some sufficient conditions of oscillation criteria for it. Some applications are also presented for the established results.

Keywords: Difference Equations, Oscillation, Fractional Order, Damping.

\section{Introduction}

Recent years have witnessed the study of qualitative properties, especially oscillation of solutions, of fractional difference equations, [3], and [7]. In this paper, we investigate the oscillatory properties a class of fractional difference equations with damping term of the form

$$
\Delta\left(c(t)\left(\Delta^{\alpha} x(t)\right)^{\gamma}\right)+p(t)\left(\Delta^{\alpha} x(t)\right)^{\gamma}+q(t)\left(\sum_{s=t_{0}}^{t-1+\alpha}(t-s-1)^{(-\alpha)} x(s)\right)^{\gamma}=0, t \in N_{t_{0}+1-\alpha}
$$

where $\Delta^{\alpha}$ denotes the Riemann-Liouville difference operator of order $0<\alpha \leq 1$ and $\gamma>0$ is a quotient of odd positive integers.

(H). $p(t)$ and $q(t)$ are positive sequences on $t_{0}>0$ and $f: R \rightarrow R$ is a continuous function and $\sum_{s=t_{0}}^{\infty} \frac{1}{\mu(s) c(s)}=\infty$.

A solution $x(t)$ of $(1)$ is said to be oscillatory if it is neither eventually positive nor eventually negative; otherwise, it is nonoscillatory. Equation (1) is said to be oscillatory if all its solutions are oscillatory.

\section{Preliminaries and basic lemmas}

In this section, we introduce some preliminary results of discrete fractional calculus, which will be used throughout this paper.

Definition 2.1: (See [8]) Let $v>0$. The $v$-th fractional sum $f$ is defined by 
$\Delta^{-v} f(t)=\frac{1}{\Gamma(v)} \sum_{s=a}^{t-v}(t-s-1)^{(v-1)} f(s)$,

Where $f$ is defined for $s \equiv a \bmod (1)$ and $\Delta^{-v} f$ is defined for $t \equiv(a+v) \bmod (1)$ and $\mathrm{t}^{(v)}=\frac{\Gamma(t+1)}{\Gamma(t-v+1)}$. The fractional sum $\Delta^{-v} f$ maps functions defined on $N_{a}$ to functions defined on $N_{a+v}$.

Definition 2.2: (see [8]) let $\mu>0$ and $m-1<\mu<m$ where $m$ denotes a positive integer $\mathrm{m}=\lceil\mu\rceil$. Set $v=m-\mu$. The $\mu-$ th fractional difference is defined as $\Delta^{\mu} f(t)=\Delta^{m-v_{f}(t)=\Delta^{m}} \Delta^{-v} f(t)$.

Lemma 2.3: Let $a \geq 0, b, X \in R$, then $b \sqrt{a} X-a X^{2} \leq \frac{b^{2}}{4}$

Lemma 2.4: Let $x(t)$ be a solution of (1) and let

$G(t)=\sum_{s=t_{0}}^{t-1+\alpha}(t-s-1)^{(-\alpha)} x(s)$

Then

$\Delta(G(t))=\Gamma(1-\alpha) \Delta^{\alpha}(x(t))$.

Proof:

$$
\begin{gathered}
G(t)=\sum_{s=t_{0}}^{t-1+\alpha}(t-s-1)^{(-\alpha)} x(s)=\sum_{s=t_{0}}^{t-(1-\alpha)}(t-s-1)^{(1-\alpha)-1} x(s) \\
=\Gamma(1-\alpha) \Delta^{-(1-\alpha)} x(t),
\end{gathered}
$$

which implies?

$\Delta(G(t))=\Gamma(1-\alpha) \Delta \Delta^{-(1-\alpha)} x(t)=\Gamma(1-\alpha) \Delta^{\alpha} x(t)$.

Now, we assume that $c(t)>p(t)$ and define a sequence

$$
\mu(t)=\prod_{s=t_{0}}^{t-1} \frac{c(s)}{c(s)-p(s)} \forall t \geq t_{0} .
$$

and

$$
\Delta \mu(t)=\frac{p(t)}{c(t)-p(t)} \mu(t) \text { and } \mu(\mathrm{t}+1)=\frac{c(t)}{c(t)-p(t)} \mu(t)
$$

\section{Main results}

Theorem 3.1: Suppose that $(H)$ holds and $\sum_{s=t_{0}}^{\infty} \frac{1}{\mu(s) c(s)}=\infty$, there exists a positive sequence $b(t)$ such that

$\limsup _{t \rightarrow \infty} \sum_{s=t_{1}}^{t-1}\left(b(s) q(s) \mu(s+1)-\frac{\left(\Delta b_{+}(s)\right)^{2}}{4 b^{2}(s+1) R(s)}\right)=\infty$,

where $\Delta b_{+}(s)=\max \{\Delta b(s), 0\}, R(t)=\frac{b(t) \Gamma(1-\alpha)^{\gamma}}{b^{2}(t+1) \mu(t) c(t)}$. Then every solution of $(1)$ is oscillatory.

Proof: Suppose that $\mathrm{x}(\mathrm{t})$ is a nonoscillatory solution of (1). Without loss of generality, we may assume that $\mathrm{x}(\mathrm{t})$ is an eventually positive of (1). Then there exists $t_{1} \geq t_{0}$ such that

$x(t)>0$ and $G(t)>0$ for $t \geq t_{1}$,

where $\mathrm{G}$ is defined as in (2). Therefore, it follows from (1) that

$$
\begin{aligned}
\Delta\left(\mu(t) c(t)\left(\Delta^{\alpha} x(t)\right)^{\gamma}\right)=\mu(t+1) \Delta\left(c(t)\left(\Delta^{\alpha} x(t)\right)^{\gamma}\right)+\left(c(t)\left(\Delta^{\alpha} x(t)\right)^{\gamma}\right) \Delta \mu(t) \\
=\mu(t+1) \Delta\left(c(t)\left(\Delta^{\alpha} x(t)\right)^{\gamma}\right)+\left(c(t)\left(\Delta^{\alpha} x(t)\right)^{\gamma}\right)\left(\frac{p(t)}{c(t)-p(t)}\right)\left(\frac{c(t)-p(t)}{c(t)}\right) \mu(t+1) \\
=\mu(t+1)\left(\Delta\left(c(t)\left(\Delta^{\alpha} x(t)\right)^{\gamma}\right)+p(t)\left(\Delta^{\alpha} x(t)\right)^{\gamma}\right)
\end{aligned}
$$

$\Delta\left(\mu(t) c(t)\left(\Delta^{\alpha} x(t)\right)^{\gamma}\right)=-\mu(t+1) q(t)(G(t))^{\gamma}<0$ for $t \geq t_{1}$ 
Thus $\left.\mu(t) c(t)\left(\Delta^{\alpha} x(t)\right)^{\gamma}\right)$ is a strictly non-increasing sequence and is eventually of one sign on $t \geq t_{1}$. First we show that $\mu(t) c(t)\left(\Delta^{\alpha} x(t)\right)^{\gamma} \quad$ is eventually positive. Suppose there is an integer $t_{1}>t_{0}$ such that $\mu(t) c(t)\left(\Delta^{\alpha} x(t)\right)^{\gamma}=\delta<0$ for $t \geq t_{1}$, so that

$\mu(t) c(t)\left(\Delta^{\alpha} x(t)\right)^{\gamma} \leq \mu\left(t_{1}\right) c\left(t_{1}\right)\left(\Delta^{\alpha} x\left(t_{1}\right)\right)^{\gamma}=\delta<0$

$\left(\Delta^{\alpha} x(t)\right)^{\gamma} \leq \frac{\delta}{\mu(t) c(t)}<0$

which implies that

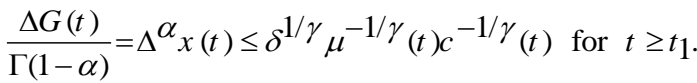

Summing both sides of the inequality (9) from $t_{1}$ to $t-1$ yields

$G(t) \leq G\left(t_{1}\right)+\Gamma(1-\alpha) \delta^{1 / \gamma} \sum_{s=t_{1}}^{t-1} \mu^{-1 / \gamma}(s) c^{-1 / \gamma}(s) \rightarrow-\infty$ as $t \rightarrow \infty$,

This contradicts the fact that $\mathrm{G}(\mathrm{t})>0$. Hence $\mu(t) c(t)\left(\Delta^{\alpha} x(t)\right)^{\gamma}>0$ is eventually positive. Define the function $\omega(t)$ by the Riccati substitution

$\omega(t)=b(t) \frac{\mu(t) c(t)\left(\Delta^{\alpha} x(t)\right)^{\gamma}}{G^{\gamma}(t)}$ for $\mathrm{t} \geq \mathrm{t}_{1}$.

Then we have $\omega(t)>0$ for $t \geq t_{1}$. It follows that

$\Delta \omega(t) \leq \Delta b_{+}(t) \frac{\omega(t+1)}{b(t+1)}-b(t) q(t) \mu(t+1)-\frac{b(t) \mu(t+1) c(t+1)\left(\Delta^{\alpha} x(t+1)\right)^{\gamma} \Delta G^{\gamma}(t)}{G^{2 \gamma}(t+1)}$.

Now using the following inequality (see [1]), we obtain

${ }_{x}^{\beta}-y^{\beta} \geq(x-y)^{\beta}$,

We have

$\mu(t) c(t)\left(\Delta^{\alpha} x(t)\right)^{\gamma} \geq \mu(t+1) c(t+1)\left(\Delta^{\alpha} x(t+1)\right)^{\gamma}$

$\left(\Delta^{\alpha} x(t)\right)^{\gamma} \geq \frac{\mu(t+1) c(t+1)}{\mu(t) c(t)}\left(\Delta^{\alpha} x(t+1)\right)^{\gamma}$.

Using the above inequality, we obtain

$\Delta \omega(t) \leq \Delta b_{+}(t) \frac{\omega(t+1)}{b(t+1)}-b(t) q(t) \mu(t+1)-\frac{b(t) \mu(t+1) c(t+1)\left(\Delta^{\alpha} x(t+1)\right)^{\gamma}(\Delta G(t))^{\gamma}}{G^{2 \gamma}(t+1)}$

$\leq \Delta b_{+}(t) \frac{\omega(t+1)}{b(t+1)}-b(t) q(t) \mu(t+1)-\frac{b(t) \Gamma(1-\alpha)^{\gamma}}{b^{2}(t+1) \mu(t) c(t)} \omega(t+1)^{2}$

$\Delta \omega(t) \leq \Delta b_{+}(t) \frac{\omega(t+1)}{b(t+1)}-b(t) q(t) \mu(t+1)-R(t) \omega(t+1)^{2}$

where $R(t)=\frac{b(t) \Gamma(1-\alpha)^{\gamma}}{b^{2}(t+1) \mu(t) c(t)}$. Take $a=R(t), b=\frac{\Delta b_{+}(t)}{b(t+1) \sqrt{R(t)}}$ and $\mathrm{X}=\omega(\mathrm{t}+1)$.

Using Lemma 2.3, we get

$\frac{\Delta b_{+}(t)}{b(t+1) \sqrt{R(t)}} \sqrt{R(t)} \omega(t+1)-R(t) \omega(t+1)^{2} \leq \frac{\left(\frac{\Delta b_{+}(t)}{b(t+1) \sqrt{R(t)}}\right)^{2}}{4}$

From (12), we conclude that

$\Delta \omega(t) \leq-b(t) q(t) \mu(t+1)+\frac{\left(\Delta b_{+}(t)\right)^{2}}{4 b^{2}(t+1) R(t)}$.

Summing the above inequality from $t_{1}$ to $t-1$, we have

$\sum_{s=t_{1}}^{t-1}\left(b(s) q(s) \mu(s+1)-\frac{\left(\Delta b_{+}(s)\right)^{2}}{4 b^{2}(s+1) R(s)}\right) \leq \omega\left(t_{1}\right)-\omega(t) \leq \omega\left(t_{1}\right)<\infty$, for $t \geq t_{1}$

Letting $t \rightarrow \infty$, we get 
$\limsup _{t \rightarrow \infty} \sum_{s=t_{1}}^{t-1}\left(b(s) q(s) \mu(s+1)-\frac{\left(\Delta b_{+}(s)\right)^{2}}{4 b^{2}(s+1) R(s)}\right) \leq \omega\left(t_{1}\right)<\infty$.

This contradicts (6). The proof is complete.

Theorem 3.2: Suppose that $(H)$ holds. Furthermore, assume that there exists a positive sequence $b(t)$ such that $H(t, t)=0$ for $t \geq t_{0} \quad H(t, s)>0 \quad t>s \geq t_{0}$ $\Delta_{2} H(t, s)=H(t, s+1)-H(t, s) \leq 0$ for $t \geq s \geq t_{0}$.

If

$\limsup _{t \rightarrow \infty} \frac{1}{H\left(t, t_{0}\right)} \sum_{s=t_{0}}^{t-1}\left(b(s) q(s) \mu(s+1) H(t, s)-\frac{h_{+}^{2}(t, s)}{4 H(t, s) R(s)}\right)=\infty$

where $h_{+}(t, s)=\Delta_{2} H(t, s)+\frac{H(t, s) \Delta b_{+}(s)}{b(s+1)}$ and $\Delta \mathrm{b}_{+}(s)=\max [\Delta b(s), 0]$. Then every solution of (1) is oscillatory.

Proof: Suppose the contrary that $x(t)$ is a nonoscillatory solution of (1). Without loss of generality, we may assume that $x(t)$ is an eventually positive solution of (1). We proceed as in the proof of Theorem (3.1) to get (12) hold.

Multiplying (12) by $H(t, s)$ and summing from $t_{1}$ to $t-1$, we obtain

$$
\begin{aligned}
\sum_{s=t_{1}}^{t-1} b(s) q(s) \mu(s+1) H(t, s) & \leq-\sum_{s=t_{1}}^{t-1} H(t, s) \Delta \omega(s)+\sum_{s=t_{1}}^{t-1} H(t, s) \Delta b_{+}(s) \frac{\omega(s+1)}{b(s+1)} \\
& -\sum_{s=t_{1}}^{t-1} H(t, s) R(s) \omega^{2}(s+1)
\end{aligned}
$$

Using summation by parts formula, we get

$$
\begin{aligned}
-\sum_{s=t_{1}}^{t-1} H(t, s) \Delta \omega(s) & =-[H(t, s) \omega(s)]_{s=t_{1}}^{t}+\sum_{s=t_{1}}^{t-1} \omega(s+1) \Delta_{2} H(t, s) \\
& =H\left(t, t_{1}\right) \omega\left(t_{1}\right)+\sum_{s=t_{1}}^{t-1} \omega(s+1) \Delta_{2} H(t, s)
\end{aligned}
$$

Now, we have

$$
\sum_{s=t_{1}}^{t-1} b(s) q(s) \mu(s+1) H(t, s) \leq H\left(t, t_{1}\right) \omega\left(t_{1}\right)+\sum_{s=t_{1}}^{t-1}\left(h_{+}(t, s) \omega(s+1)-H(t, s) R(s) \omega^{2}(s+1)\right)
$$

where $h_{+}(t, s)=\Delta_{2} H(t, s)+\frac{H(t, s) \Delta b_{+}(s)}{b(s+1)}$.

Taking $a=H(t, s) R(s), b=\frac{h_{+}(t, s)}{\sqrt{H(t, s) R(s)}}$ and $\mathrm{X}=\omega(\mathrm{t}+1)$ and using the Lemma 2.3, we get

$\frac{h_{+}(t, s)}{\sqrt{H(t, s) R(s)}} \sqrt{H(t, s) R(s)} \omega(t+1)-H(t, s) R(s) \omega(t+1)^{2} \leq \frac{\left(\frac{h_{+}(t, s)}{\sqrt{H(t, s) R(s)}}\right)^{2}}{4}$

We have $0<H\left(t, t_{1}\right) \leq H\left(t, t_{0}\right)$ for $t>t_{1} \geq t_{0}$, from equation (16),

$$
\begin{aligned}
\sum_{s=t_{1}}^{t-1} b(s) q(s) \mu(s+1) H(t, s) \leq H\left(t, t_{1}\right) \omega\left(t_{1}\right)+ & \sum_{s=t_{1}}^{t-1} \frac{h_{+}^{2}(t, s)}{4 H(t, s) R(s)} \\
\sum_{s=t_{1}}^{t-1}\left(b(s) q(s) \mu(s+1) H(t, s)-\frac{h_{+}^{2}(t, s)}{4 H(t, s) R(s)}\right) & \leq H\left(t, t_{1}\right) \omega\left(t_{1}\right) \\
& \leq H\left(t, t_{0}\right) \omega\left(t_{1}\right) .
\end{aligned}
$$

Since $0<H(t, s) \leq H\left(t, t_{0}\right)$ for $t>s \geq t_{0}$, we have $0<\frac{H(t, s)}{H\left(t, t_{0}\right)} \leq 1$ for $t>s \geq t_{0}$. Hence it follows that 


$$
\begin{aligned}
& \frac{1}{H\left(t, t_{0}\right)} \sum_{s=t_{0}}^{t-1}\left(b(s) q(s) \mu(s+1) H(t, s)-\frac{h_{+}^{2}(t, s)}{4 H(t, s) R(s)}\right)= \\
& \frac{1}{H\left(t, t_{0}\right)} \sum_{s=t_{0}}^{t_{1}-1}\left(b(s) q(s) \mu(s+1) H(t, s)-\frac{h_{+}^{2}(t, s)}{4 H(t, s) R(s)}\right) \\
& +\frac{1}{H\left(t, t_{0}\right)} \sum_{s=t_{1}}^{t-1}\left(b(s) q(s) \mu(s+1) H(t, s)-\frac{h_{+}^{2}(t, s)}{4 H(t, s) R(s)}\right) \\
& \leq \frac{1}{H\left(t, t_{0}\right)}{ }_{s=t}^{t} \sum_{s}^{-1} b(s) q(s) \mu(s+1) H(t, s)+\omega\left(t_{1}\right) \\
& \leq \sum_{s=t_{0}}^{t_{1}-1} b(s) q(s) \mu(s+1)+\omega\left(t_{1}\right)
\end{aligned}
$$

Letting $t \rightarrow \infty$, we have

$$
\begin{gathered}
\limsup _{t \rightarrow \infty} \frac{1}{H\left(t, t_{0}\right)} \sum_{s=t_{0}}^{t-1}\left(b(s) q(s) \mu(s+1) H(t, s)-\frac{h_{+}^{2}(t, s)}{4 H(t, s) R(s)}\right) \\
\leq \sum_{s=t_{0}}^{t_{1}-1} b(s) q(s) \mu(s+1)+\omega\left(t_{1}\right)<\infty,
\end{gathered}
$$

This is a contradiction to (13). The proof is complete.

Example 3.3: Consider the fractional difference equation

$$
\begin{aligned}
& \Delta\left(\Delta^{\alpha} x(t)\right)+\frac{1}{t+1} \Delta^{\alpha} x(t)+\frac{1}{t+1} \sum_{s=t_{0}}^{t-1+\alpha}(t-s-1)^{(-\alpha)} x(s)=0, \\
& \text { where } \alpha=0.5, \gamma=1, c(t)=1, p(t)=\frac{1}{t+1} \text { and } q(t)=\frac{1}{t+1} \text {. Since } \\
& \mu(\mathrm{t})=\prod_{s=1}^{t-1} \frac{1}{1-\frac{1}{s+1}}=t .
\end{aligned}
$$

we find that $(\mathrm{H})$ holds. We will apply Theorem (3.1) and it remains to show condition (6) is satisfied. Taking $b(s)=s$, we obtain

$$
\limsup _{t \rightarrow \infty} \sum_{s=t}^{t-1}\left(b(s) q(s) \mu(s+1)-\frac{\left(\Delta b_{+}(s)\right)^{2}}{4 b^{2}(s+1) R(s)}\right)=\limsup _{t \rightarrow \infty} \sum_{s=t}^{t-1}\left(s-\frac{1}{4 \sqrt{\pi}}\right)=\infty
$$

which implies that (6) holds. Therefore, by Theorem (3.1) every solution of (17) is oscillatory.

\section{References}

[1] G.H. Hardy, J.E. Littlewood, G. Polya, Inequalities, Cambridge University Press, Cambridge (1959)

[2] Ravi P. Agarwal, Martin Bohner, Said R. Grace, Donal O Regan, Discrete Oscillation Theory, Hindawi Publishing Corporation, 2005.

[3] Said R. Grace, Ravi P. Agarwal, Patricia J.Y. Wong, Agacik Zafer, On the oscillation of fractional differential equations, FCAA, Vol15, No.2 (2012).

[4] Da-Xue Chen, Oscillatory behavior of a class of fractional differential equations with damping, U.P.B. Sci. Bull., Series A, Vol. 75, Iss. 1, 2013.

[5] S.Lourdu Marian, M. Reni Sagayaraj, A.George Maria Selvam, M.Paul Loganathan, Oscillation of fractional nonlinear difference equations, Mathematica Aeterna, Vol. 2, 2012, no. 9, 805 - 813.

[6] M. Reni Sagayaraj, A.George Maria Selvam, M.Paul Loganathan, Oscillation of Caputo like discrete fractional difference equations, IJPAM, Vol. 89, no. 5,2013, $667-677$.

[7] M. Reni Sagayaraj, A.George Maria Selvam, M.Paul Loganathan, On the oscillation nonlinear fractional nonlinear difference equations, Mathematica Aeterna, Vol. 4, 2014, no. 1, 91 - 99.

[8] F. M. Atici, P. W. Eloe, Initial value problems in discrete fractional calculus, Proceedings of the American Mathematical Society, Vol. 137, No. 3, pp. 981-989, 2009.

[9] George A.Anastassiou, Discrete fractional calculus and inequalities, arXiv 0911.3370v1 [math CA] 17 Nov 2009. 